\title{
Experiencia pedagógica en la implementación de la clase invertida en el proceso de formación académica
}

\author{
Zeidy Sandra López Collazo, Andrés Rodríguez Jiménez, Yosdey Dávila Valdés \\ Universidad de Artemisa, Código Postal: 33700, Cuba
}

DOI: https://doi.org/10.33017/RevECIPeru2018.0020/

\section{Resumen}

El presente artículo revela la experiencia pedagógica en la implementación de la metodología de Flipped Classroom o clase invertida en la formación académica de la segunda edición del programa de la Maestría en Didáctica que se desarrolla en la Universidad de Artemisa, Cuba. Todo ello sustentado en la aplicación de métodos del nivel teórico que permitieron sistematizar los referentes teórico-metodológicos acerca de la Flipped Classroom. Esta implementación se desarrolló en el curso de Metodología de la Investigación Educativa, particularmente en el tema correspondiente a La tesis como un tipo de documento científico, Para ello se dispuso un material audiovisual que los aspirantes debían visualizar independientemente, con la ayuda de una guía de observación que se les facilitó. La valoración del impacto sobre el proceso de enseñanza-aprendizaje se realizó mediante una entrevista grupal en la que todos los aspirantes pudieron expresar abiertamente, ante el colectivo de aspirantes y profesores, sus opiniones y criterios en relación con la metodología empleada. También se aplicó una encuesta para que cada estudiante evaluara sus niveles de satisfacción en este sentido. A partir de las respuestas a dicha encuesta se determinaron las medianas de los diferentes indicadores explorados mediante la estadística inferencial y el empleo del Coeficiente de Correlación Multidimensional rpj, como procedimiento lógico aceptado por la Teoría Clásica de los Test para determinar el grado de objetividad de los criterios emitidos. Los resultados obtenidos evidencian una tendencia a elevados niveles de satisfacción en los diferentes aspectos explorados.

Descriptores: Aprendizaje inverso, clase invertida, integración, aprendizaje audiovisual

\begin{abstract}
This article reveals the pedagogical experience in the implementation of the Flipped Classroom methodology or inverted class in the academic formation of the second edition of the Master's program in Didactics that takes place at the University of Artemisa, Cuba. All this is based on the application of theoretical level methods that allowed systematizing the theoretical-methodological references about the Flipped Classroom. This implementation was developed in the course of Educational Research Methodology, particularly in the topic corresponding to the thesis as a type of scientific document, for which an audiovisual material was available that the candidates had to visualize independently, with the help of a guide of observation that was provided. The assessment of the impact on the teaching-learning process was carried out through a group interview in which all the applicants were able to openly express, before the group of aspirants and professors, their opinions and criteria in relation to the methodology used. A survey was also applied so that each student could evaluate their levels of satisfaction in this regard. Based on the responses to this survey, the medians of the different indicators explored through inferential statistics and the use of the Multidimensional Correlation Coefficient rpj were determined, as a logical procedure accepted by the Classical Test Theory to determine the degree of objectivity of the criteria issued. The results obtained show a tendency to high levels of satisfaction in the different aspects explored.
\end{abstract}

Keywords: Flip teaching, Flipped Classroom, integration, audiovisual learning. 


\section{Introducción}

La sociedad cubana actual requiere de profesionales de la educación versátiles, en pos de una mejora en la calidad de la educación ofertada. Es por ello que el programa de Maestría en Didáctica que se desarrolla actualmente en la Universidad de Artemisa de Cuba, atiende una necesidad social y académica, se encuentra concebido para que los maestrantes logren gestionar el conocimiento con el fin que se apropien activa y creadoramente del empleo de nuevos métodos y técnicas de enseñanza y de investigación. El ritmo de desarrollo de la provincia de Artemisa estará condicionado por el nivel que logre alcanzar en la formación de las actuales y futuras generaciones de artemiseños. Para ello se requiere elevar a planos superiores de calidad, la clase.

Esta exigencia se manifiesta tanto en los en los diferentes niveles de enseñanza como en la formación de los profesionales que egresan de las instituciones de nivel superior de la provincia. El programa se inscribe en la concepción general del sistema educacional cubano, como parte del cuarto nivel de educación y es asumido y desarrollado como nuevo paradigma de superación en lo teórico y en lo metodológico. Tiene como sustento esencial la experiencia profesional y los estudios teóricosprácticos sobre problemas de la educación.

El modelo del programa de formación académica obedece al denominado modelo tradicional de educación que se viene realizando desde hace décadas, el cual se desarrolla en la modalidad presencial tradicional con una frecuencia semanal en la que se imparte el sistema de conocimientos de los diversos cursos que conforman el currículo todo lo cual demanda la sistematización, la profundización y la actualización de los aspirantes en el horario no presencial. Es por ello que el claustro de profesores del programa académico referido está haciendo un esfuerzo por introducir nuevos métodos e implementar novedosas herramientas al proceso de enseñanza-aprendizaje.

Por lo tanto, se trataría de implementar metodologías docentes en las que se integren los conocimientos adquiridos en la adecuada proporción para maximizar tanto la adquisición de los objetivos de aprendizaje como la gestión del conocimiento. Se trata de mejorar, facilitar y potenciar la adquisición y apropiación del conocimiento con ayuda de las Tecnologías de la
Información y la Comunicación (TIC) sin abandonar aquellas herramientas tradicionales que dotan a los profesores de buenas cualidades profesionales. Este equilibrio entre metodologías significa un reto importante para el claustro de profesores del programa académico de Maestría en Didáctica.

Sobre este particular se ha discutido mucho acerca de la flexibilización de los espacios y tiempos en los que los maestrantes pueden dedicarse a trabajar sus materias o profundizar en ellas. Prácticamente todos ellos disponen de dispositivos móviles, los cuales son capaces de almacenar gran cantidad de información y les permite disponer de libros y material de estudio a cualquier hora y en cualquier lugar.

En este sentido recientemente se ha producido un incremento considerablemente de los espacios con disponibilidad de Wifi en el territorio cubano permitiendo la transmisión de información y la comunicación prácticamente instantánea, todo lo cual hace posible que los maestrantes puedan trabajar en grupos y colaborar en la resolución de tareas a pesar de encontrarse a grandes distancias unos de otros y del centro universitario. El uso generalizado de las TIC en los últimos años y su integración progresiva en las aulas ha conducido hacia un cambio de metodologías de los profesores, que ha visto en ellas una poderosa herramienta para potenciar el aprendizaje y su influencia en la motivación de los aspirantes.

Estos cambios no son fáciles de realizar y requieren un gran esfuerzo por parte del claustro de profesores para transformar una clase presencial tradicional en una clase invertida o Flipped Classroom. Es interesante comprobar cómo la Flipped Classroom ofrece una reestructuración del trabajo en el aula que permite un mayor aprovechamiento del tiempo para poder maximizar, por un lado, el seguimiento de tareas y la resolución de dudas por parte del profesor, y por otro, incentivar el trabajo autónomo y cooperativo de los aspirantes.

El término Flipped Classroom fue acuñado por Bergmann y Sams (2012) [1], dos profesores que empezaron a grabar y distribuir vídeos de sus lecciones para ayudar a aquellos de sus estudiantes que faltaban a clase por cualquier motivo. Mediante la puesta en marcha de esta idea, se dieron cuenta de que además de facilitar el estudio a dichos estudiantes, estaban consiguiendo tener más tiempo para responder a las necesidades 
educativas de cada estudiante, por lo que terminaron haciendo la propuesta ante la cual se adscriben los autores y sustentan teóricamente la experiencia pedagógica implementada.

Siguiendo a los profesores referidos, se puede apreciar que en lo que básicamente consiste invertir la clase es en llevar a cabo lo que normalmente se hace en clase a casa, y a clase lo que tradicionalmente se hace en casa como deberes. Pero advierten que en el fondo clase invertida es mucho más que eso, y puede tener múltiples variantes. Una de las dinámicas que proponen estos autores es comenzar la clase con unos cuantos minutos de debate sobre el vídeo que los estudiantes debieron visualizar anteriormente en sus casas o en cualquier otro lugar.

Al respecto García (2013) [2] insistió en plantear que el material teórico debe ser proporcionado por el profesor en formato digital, formato de video, y la responsabilidad de trabajarlo recae sobre el estudiante, mientras que el papel del profesor en la clase invertida es más exigente que en la tradicional.

El empleo de la Flipped Classroom representa la metodología adecuada para adquirir y apropiarse de los conocimientos de manera integrada. Opazo, Acuña, Rojas, (2016) [3]. Por todo ello el objetivo del artículo que se presenta está dirigido a socializar la experiencia pedagógica en la implementación de la metodología de Flipped Classroom o clase invertida en la formación académica de la segunda edición del programa de la Maestría en Didáctica que se desarrolla en la Universidad de Artemisa, particularmente en el curso de Metodología de la Investigación Educativa.

\section{Metodología}

La implementación de la experiencia pedagógica se realiza con el grupo de aspirantes a máster, el cual está conformado por una matrícula de 38 y para los efectos del estudio, la muestra se constituyó por los 30 que del grupo respondieron la encuesta de satisfacción aplicada.

Como trabajo previo a la implementación de la clase invertida, se preparó un material audiovisual en el que los profesores del curso de Metodología de la Investigación Educativa, empleando la aplicación TechSmith Camtasia Studio v8.6.0 Build 2079, explican sobre la base de una presentación de Power point, los aspectos esenciales relacionados con la elaboración de la tesis como documento científico.

Este colectivo de profesores confeccionó también orientaciones para la observación individual del audiovisual por parte de los aspirantes. Para llevar a cabo la clase invertida en la clase previa, con una semana de antelación a la clase presencial, se les explicó a los aspirantes en qué consistía básicamente esta metodología, destacando la necesidad de su preparación previa para desarrollar la sesión presencial en la siguiente clase. En esta oportunidad se motivó a los aspirantes acerca de la temática que sería tratada y se les entregó el material audiovisual previamente elaborado, acompañado de las orientaciones para su visualización.

El contenido de las orientaciones para la observación del material audiovisual tiene como objetivo orientar a los aspirantes durante la visualización acerca del aprendizaje que debe realizar mediante una relación de preguntas que deben responder por escrito y a la vez se les estimula a que anoten las dudas que les queden, después de la visualización del material y a elaborar preguntas acerca del contenido del audiovisual. Las propuestas de preguntas a responder individualmente, en todos los casos, están dirigidas al vencimiento por los aspirantes de los niveles más bajos de asimilación del conocimiento (comprensión y repetición).

Durante la fase presencial se realizó una sesión de aprendizaje cooperativo, que partió de la socialización de las respuestas a las preguntas construidas individualmente antes de la actividad presencial y que continuó con la solución por equipos de situaciones de aprendizaje propuestas por los profesores. La actividad presencial posibilitó el trabajo cooperativo para discutir las respuestas a las preguntas orientadas y para solucionar situaciones de aprendizaje que requirieron niveles de asimilación más complejos, pues en estas condiciones cada estudiante pudo contar con la ayuda de otros colegas más aventajados y con la del profesor.

Al finalizar la actividad presencial se realizó una entrevista grupal, dirigida esencialmente a que los aspirantes expresaran ante el colectivo de colegas y de profesores lo que consideraron como positivo y como negativo en la implementación de la metodología de la clase invertida.

La encuesta de satisfacción es aplicada a los aspirantes una vez implementada, en sus dos fases, 
la metodología de la clase invertida. El cuestionario fue elaborado por los autores. Los aspectos a evaluar por los aspirantes están formulados de forma tal que si el sujeto los evalúa con valores entre 4 (bastante de acuerdo) o 5 (muy de acuerdo), se puede inferir que la experiencia tuvo un efecto positivo sobre su aprendizaje, pues como se ha establecido en la introducción de este trabajo, estos se refieren a atributos que fundamentan la utilidad del empleo de la metodología de la clase invertida. La única excepción en este sentido lo constituye el aspecto 16 de la encuesta (Prefiero el sistema tradicional de enseñanza, en el que el profesor explica los contenidos en clase, aunque se dedique un menor tiempo a realizar actividades colaborativas), que como se puede apreciar, una evaluación de 4 o 5 en él, implicaría que el estudiante rechaza el empleo de la metodología de la clase invertida.

La encuesta se aplicó una semana después de realizar la actividad presencial relacionada con la temática y los resultados se procesaron empleando el Coeficiente de Correlación Multidimensional rpj, como procedimiento lógico aceptado por la Teoría Clásica de los Test para determinar el grado de objetividad de los criterios emitidos. Pérez (2015) [4], que posibilitó obtener el valor de mediana para cada uno de los aspectos evaluados y la determinación de la tendencia central en cada uno de ellos.

\section{Resultados}

\subsection{Diseño de la tarea previa}

Se partió de las tres consideraciones que deben tenerse en cuenta: para qué se hace la tarea previa, con qué materiales se debe llevar a cabo y cómo se ha de hacer Marqués (2016) [5].

En relación con para qué se hace, es claro que tanto para los aspirantes, como para los profesores resulta de importancia capital, que los aspirantes demuestren sus competencias en la elaboración de la tesis que presentarán en su defensa de la maestría, lo que constituye un importante motor motivacional para cumplir con la tarea previa.

La definición de con qué materiales llevar a cabo la tarea previa reportó dos resultados: un material audiovisual y unas orientaciones concretas para su visualización de manera independiente.

El audiovisual es un material de 24 minutos en que se combina una presentación de power point con la explicación de dos profesores, para trasmitir algunos conocimientos esenciales que requieren asimilar los aspirantes para la elaboración de su tesis de maestría: tipos de tesis, momentos por lo que transita (redacción y defensa), algoritmo general para la redacción, requisitos generales de una tesis (de forma y de contenido), estructura de una tesis (título, introducción, fundamentos teóricos, aspectos metodológicos de la investigación, resultados y discusión, conclusiones y recomendaciones, bibliografía y anexos).

Las orientaciones elaboradas se estructuraron en dos partes fundamentales: indicaciones generales para operar con el audiovisual y 16 preguntas a responder por escrito a partir de la visualización realizada. Este material que a continuación se presenta está dirigido a dar respuesta a la interrogante ¿cómo se hace la tarea previa?

Observe con detenimiento el video que se ofrece acerca de la memoria escrita de la tesis de maestría y:

I. Responde por escrito con la mayor precisión posible cada una de las preguntas que se formulan. II. Anote cada una de las dudas que te queden acerca de los contenidos tratados en el video.

III. Elabora una pregunta relacionada con el contenido tratado que no te haya quedado suficientemente claro en el video observado.

\section{Preguntas a responder por escrito}

1. ¿Qué es una tesis y por qué momentos transcurre?

2. ¿Por qué es importante que la tesis esté correctamente redactada?

3. ¿Cuáles son los pasos que constituyen el algoritmo general para la redacción? ¿A qué parte de la tesis se aplican estos pasos?

4. ¿Por qué es importante que el aspirante sea receptivo a los señalamientos que se le realizan durante la elaboración de la memoria escrita?

5. Describa cómo se cumplen cada uno de los requisitos de contenido a través de la tesis

6 . Mencione al menos tres requisitos que debe cumplir el lenguaje que se emplea en la redacción de la tesis

7. Describa cómo se emplean las siglas a través de la redacción de la tesis

8. Mencione los componentes que conforman el cuerpo de la tesis cuál o cuáles de ellos no tienen necesariamente que estar presentes.

9. Mencione los elementos que conforman la portada de una tesis

10. ¿A quiénes se dirigen fundamentalmente los agradecimientos en una tesis?

11. Caracterice la síntesis de una tesis

12. Describa cómo se colocan los títulos de los sub epígrafes en el índice de una tesis 
13. ¿Qué se página necesariamente en la tesis y cómo?

14. Mencione dos sugerencias que ayudan a acortar el número de palabras en el título.

15. Analice el título que aparece en el ejemplo de portada que se presenta en el video y determine si cumple con los requisitos establecidos o no y por qué

16. ¿Por qué se debe poner especial énfasis en la redacción de la introducción de la tesis?

Este material guía claramente a los aspirantes en la elaboración de un producto de la tarea previa realizada: la respuesta por escrito a las 16 preguntas formuladas. Además, se les estimula a que identifiquen sus dudas y a que formulen preguntas a las que la visualización del audiovisual no les permite responder.

\subsection{Diseño de la clase presencial}

Se comenzó por un intercambio general con el grupo acerca de ¿Qué tiempo les requirió realizar la tarea previa? ¿Les resultó difícil realizarla? El tiempo fue variable entre los aspirantes, pero hubo consenso en que una semana fue suficiente para cumplir con la tarea, a pesar de que todos los aspirantes son trabajadores y requirió sacrifico de su tiempo libre para realizarla.

Hubo consenso también en que fue realizable para todos. La recogida de esta información es muy valiosa, pues permite inferir que la tarea previa estuvo bien concebida y planificada, dadas las características de los aspirantes y del contexto.

A continuación, se puso en común las respuestas a las preguntas de la tarea previa, mediante un trabajo cooperativo en pequeños grupos. Ello posibilitó que se corrigieran los errores que algunos habían cometido en sus respuestas individuales y fue una forma de que cada estudiante pudiera autoevaluar su desempeño y a la vez, recibiera la evaluación de sus compañeros de grupo, como procedimientos importantes de la evaluación formativa Pérez y Taberno (2008) [6].

Esta parte inicial de la actividad presencial concluyó con una discusión en plenario de cada una de las respuestas a las preguntas lo que le posibilitó al profesor profundizar en determinados aspectos de interés relacionados con la temática, para que ya los aspirantes estuvieran preparados a partir de la realización de la tarea previa.

La clase presencial continuó con la solución, en los pequeños grupos de trabajo, de determinadas situaciones de aprendizaje, conectadas con la tarea previa, pero sin repetir, que demandaban de los aspirantes niveles más complejos de asimilación que los que tuvieron que emplear en la tarea previa.

Un ejemplo de situación de aprendizaje desarrollada por todos los pequeños grupos consistió en que se proporcionaron varios títulos de diferentes tesis de maestría ya defendidas, para que se valorara si cada uno de los títulos cumplía con los requisitos estudiados y se propusieran posibles cambios para que se cumplieran dichos requisitos.

De esta manera a los aspirantes se les exigió un cambio desde el nivel reproductivo que emplearon para solucionar la tarea previa, hacia niveles productivos en que tenían que aplicar los conocimientos adquiridos a una situación práctica vinculada con el tema objeto de estudio.

La solución de esta tarea requiere mayores esfuerzos intelectuales por parte de cada estudiante, pero a la vez cuenta con la presencia del profesor y otros colegas, que les pueden brindar la ayuda necesaria para alcanzar la solución. En esta segunda parte también se aplicaron los procedimientos de la evaluación formativa ya mencionados que realizan valiosos aportes al aprendizaje de los aspirantes.

La clase presencial concluyó con una entrevista grupal para valorar los aspectos positivos y negativos de la metodología de la clase invertida empleada, en su comparación con la metodología tradicional, en que el profesor expone de forma presencial, las esencialidades de un tema determinado y deja como tarea ejercicios en que es necesario aplicar esos conocimientos.

Los criterios emitidos por los aspirantes coincidieron en que se sintieron más cómodos en el aprendizaje empleando esta metodología porque pudieron ver el material audiovisual las veces que lo necesitaron para responder las preguntas, que pudieron responder las preguntas sin mayores tropiezos y el trabajo cooperativo en el aula les dio más confianza para corregir los errores que cometieron así como para solucionar los ejercicios de mayor complejidad que se les presentó en la clase presencial, en la que tuvieron más tiempo para profundizar y aclarar dudas que en la clase en la que se dedica la mayor parte del tiempo el profesor lo utiliza para explicar los conocimientos esenciales.

No plantearon algún aspecto negativo en comparación con la metodología tradicional de 
desarrollar las clases. Estas opiniones de los aspirantes reflejan varias potencialidades de la clase invertida:

- Los aspirantes construyen sus conocimientos a su ritmo propio, mientras que en la metodología tradicional tienen que hacerlo al ritmo que impone el grupo y el profesor y muchas veces se quedan detrás.

- Los niveles de asimilación reproductivos del conocimiento lo alcanzan de forma independiente, fuera del aula, sin mayores tropiezos.

- Los niveles de asimilación productivos que resultan de mayor complejidad se trabajan en la clase presencial donde se puede contar con la ayuda del profesor y de otros aspirantes.

- Se dispone de más tiempo en el aula para trabajar cooperativamente en pequeños grupos, en los que está presente la ayuda del profesor y de otros aspirantes para profundizar en el conocimiento, corregir errores y aclarar dudas.

\subsection{Aplicación a los aspirantes de una encuesta de satisfacción}

\subsubsection{Encuesta de satisfacción}

En relación con la integración de las TIC al proceso de enseñanza-aprendizaje, particularmente en la modalidad a distancia, concretada en la observación del video sobre redacción de la tesis de maestría, en el que usted experimentó nuevas vivencias, le pedimos emita sus criterios y opiniones, teniendo en cuenta los aspectos que se relacionan a continuación. Su colaboración será muy importante para el perfeccionamiento a realizar en ediciones futuras.

Seleccione un número de la escala que se muestra para evaluar el grado en que usted concuerda o no con cada planteamiento, en relación con el desarrollo del curso y el impacto que tuvo en su formación permanente como profesor.

\begin{tabular}{|l|l|}
\hline \multicolumn{1}{|c|}{ Aspectos } & Evaluación \\
\hline $\begin{array}{l}\text { 1. El tiempo en el aula puede ser } \\
\text { utilizado de forma más efectiva y } \\
\text { creativa }\end{array}$ & \\
\hline $\begin{array}{l}\text { 2. Es un buen complemento a la } \\
\text { clase presencial }\end{array}$ & \\
\hline $\begin{array}{l}\text { 3. Es un recurso acorde con las } \\
\text { exigencias de la sociedad actual }\end{array}$ & \\
\hline $\begin{array}{l}\text { 4. Es un material flexible que se } \\
\text { puede adaptar a las necesidades } \\
\text { de cada persona }\end{array}$ & \\
\hline
\end{tabular}

\begin{tabular}{|c|c|}
\hline $\begin{array}{l}\text { 5. Produce importantes ahorros en } \\
\text { tiempo lectivo }\end{array}$ & \\
\hline $\begin{array}{l}\text { 6. La clase en el aula se } \\
\text { "humaniza" }\end{array}$ & \\
\hline $\begin{array}{l}\text { 7. Ayuda en la consecución de } \\
\text { mayores niveles de logro, interés y } \\
\text { compromiso de los aspirantes }\end{array}$ & \\
\hline $\begin{array}{l}\text { 8. El estudiante se convierte en el } \\
\text { verdadero protagonista de su } \\
\text { aprendizaje }\end{array}$ & \\
\hline $\begin{array}{l}\text { 9. Fomenta el trabajo autónomo y } \\
\text { contribuye a una adecuada gestión } \\
\text { del tiempo }\end{array}$ & \\
\hline $\begin{array}{l}\text { 10. Posibilita su explotación en los } \\
\text { años sucesivos }\end{array}$ & \\
\hline $\begin{array}{l}\text { 11. Las clases son más prácticas } \\
\text { y tienen más trabajo colaborativo }\end{array}$ & \\
\hline $\begin{array}{l}\text { 12. Me ha permitido tener una } \\
\text { relación más cercana con los } \\
\text { profesores del curso de MIE }\end{array}$ & \\
\hline $\begin{array}{l}\text { 13. Me ha permitido tener una } \\
\text { relación más cercana con mis } \\
\text { compañeros }\end{array}$ & \\
\hline $\begin{array}{l}\text { 1. Ha aumentado mi interés por } \\
\text { el curso de MIE }\end{array}$ & \\
\hline $\begin{array}{llr}15 . ~ H a & \text { contribuido a que } \\
\text { gestione mejor mi tiempo de } \\
\text { estudio }\end{array}$ & \\
\hline $\begin{array}{l}\text { 16. Prefiero el sistema tradicional } \\
\text { de enseñanza, en el que el } \\
\text { profesor explica los contenidos } \\
\text { en clase, aunque se dedique un } \\
\text { menortiempo a realizar actividades } \\
\text { colaborativas }\end{array}$ & \\
\hline $\begin{array}{l}\text { 17. Me ha permitido reproducir } \\
\text { tantas veces como desee sin } \\
\text { importar el lugar desde el que se } \\
\text { realice }\end{array}$ & \\
\hline $\begin{array}{l}\text { 18. Me ha permitido detener la } \\
\text { explicación cuando es necesario }\end{array}$ & \\
\hline $\begin{array}{l}\text { 19. Me ha facilitado la explicación } \\
\text { de conceptos }\end{array}$ & \\
\hline $\begin{array}{l}20 . \text { Me ha posibilitado la } \\
\text { recuperación de las clases } \\
\text { perdidas, al menos de forma } \\
\text { parcial }\end{array}$ & \\
\hline
\end{tabular}

Para el procesamiento de la información se consideró la siguiente escala: 1. En desacuerdo; 2. Muy poco de acuerdo; 3. De acuerdo; 4. Bastante de acuerdo; 5. Muy de acuerdo

El Gráfico 1 muestra los resultados obtenidos para las medianas de cada uno de los ítems incluidos en el cuestionario de la encuesta de satisfacción. 


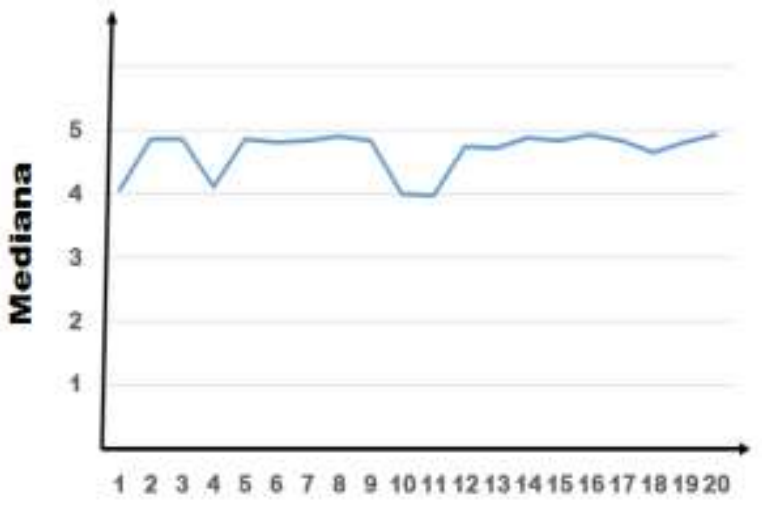

Indicadores

Gráfico 1. Valores de mediana determinados para cada uno de los indicadores incluidos en la encuesta de opinión aplicada a los aspirantes a máster.

Como se puede apreciar todos los aspectos evaluados muestran medianas iguales o superiores a 4, de lo que se infiere una influencia positiva de la experiencia pedagógica realizada sobre el aprendizaje de los aspirantes. La única excepción lo constituye el indicador 16 (Prefiero el sistema tradicional de enseñanza, en el que el profesor explica los contenidos en clase, aunque se dedique un menor tiempo a realizar actividades colaborativas), en el que la mediana cercana a 5, indica que la mayoría de los aspirantes continúan prefiriendo la metodología tradicional respecto a la de la clase invertida.

Ello resulta contradictorio con la evaluación del resto de los indicadores que indican una tendencia hacia la preferencia por la metodología de la clase invertida. Se considera, a partir de los resultados que los aspirantes no evaluaron conscientemente este indicador y siguieron la tendencia con que evaluaron al resto de los ítems propuestos, sin considerar que este aspecto debió evaluarse con el valor más bajo de la escala.

\section{Conclusiones}

La influencia positiva del empleo de la metodología de la clase invertida sobre el aprendizaje de los aspirantes que se infiere a partir de esta experiencia pedagógica coincide con resultados obtenidos por otros investigadores en el ámbito internacional: Pierce y Fox (2012) [7] afirman que lograron mayor desempeño en las evaluaciones; reportan un mejor desempeño en resolución de problemas y trabajo en equipo; Ros y García (2014) [8] entre los beneficios reportan que en contextos extra clase, se logra la participación más activa del estudiante, una mayor autonomía de trabajo y mejor gestión del tiempo.
Otros estudios han encontrado incrementos en la asistencia a clase, la motivación para aprender, y el compromiso del estudiante Grasman, Largo, \& Schmidt (2012), McLaughlin (2014); Pierce \& Fox (2012) todos ellos citados por Braseby (2014) [9]. Ello constituye una evidencia de la necesidad de emplear con mayor frecuencia y en todas las asignaturas la metodología Flipped Classroom.

\section{Referencias}

[1] O. Bergmann, J. Sams y A. Flip, Your Classroom: Talk To Every Student In Every Class Every Day (2012), pp. 62-66.

[2] A. García, El aula inversa: cambiando la respuesta a las necesidades de los estudiantes (2013), pp. 81-87.

[3] A. Opazo, J. Acuña y M. Rojas, Evaluación de metodología Flipped Classroom: primera experiencia (2016), pp. 90-99.

[4] O. Pérez, en Memorias Congreso Internacional Pedagogía, 2015, ISBN: 978959-18-1099-1.

[5] M. Marqués, Qué hay detrás de la clase al revés (Flipped Classroom), (2016), pp. 77-84.

[6] A. Pérez, B. Tabernero, Evaluación formativa y compartida en la docencia universitaria y el Espacio Europeo de Educación Superior: cuestiones clave para su puesta en práctica, Revista de Educación 347 (2008) 435-451.

[7] R. Pierce, J. Fox, Vodcasts and active-learning exercises in a "flipped classroom" model of a renal pharmacotherapy module. American Journal of Pharmaceutical Education 76 (2012) artículo 196

[8] A. Ros, A. García, Uso del vídeo docente para la clase invertida: evaluación, ventajas e inconvenientes (ACCI, 2014), pp. 423-441.

[9] A. Braseby, The Flipped Classroom idea (IDEA Center, Inc. 2014), Idea paper 57

E-mail: zeidysandra@uart.edu.cu 